\title{
A management strategy that controls lower airway eosinophilic inflammation and symptoms reduced exacerbations in asthma
}

Green RH, Brightling CE, McKenna S, et al. Asthma exacerbations and sputum eosinophil counts: a randomised controlled trial. Lancet 2002;360:1715-21.

\section{QUESTION: In patients with asthma, is a management strategy that controls lower airway eosinophilic inflammation and symptoms more effective than standard care for reducing asthma exacerbations?}

\section{Design}

Randomised \{allocation concealed*\}†, blinded (clinicians and patients),* controlled trial with 12 months of follow up.

\section{Setting \\ 3 specialist clinics at a hospital in Leicester, UK.}

\section{Patients}

74 patients (54\% men, age range $18-75$ y) who had moderate to severe asthma and probably needed continued hospital follow up. Exclusion criteria included current smokers, a smoking history of $>15$ pack years, clinically important comorbidity, poor compliance with treatment, aggravating factors that were inadequately controlled (eg, rhinitis), and a severe exacerbation within 4 weeks of entry to the trial. Follow up was $92 \%$.

\section{Intervention}

37 patients each were allocated to management with reference to the induced sputum eosinophil count (eosinophil group) or management by a modified version of the British Thoracic Society guidelines (BTS group). In the eosinophil group, decisions about anti-inflammatory treatment were made in accordance with an algorithm based on maintenance of a sputum eosinophil count $<3 \%$ with a minimum dose of anti-inflammatory treatment. In the BTS group, treatment decisions were based on usual assessments of symptoms, peak expiratory flow, and use of $\beta_{2}$ agonists.

\section{Main outcome measures}

Number of severe asthma exacerbations (defined as a decrease in the morning peak expiratory flow to $>30 \%$ below the baseline value on $\geq 2$ consecutive days or deterioration in symptoms needing treatment with oral corticosteroids) and control of eosinophil airway inflammation measured by the induced sputum eosinophil count.

\section{Main results}

Analysis was by intention to treat. Over 12 months, the number of severe exacerbations was lower in the eosinophil group than in the BTS group (35 $v 109$ total exacerbations, $\mathrm{p}=0.01$ ). The sputum eosinophil count was $63 \%$ (95\% CI 24 to 100, $\mathrm{p}=0.002$ ) lower in the eosinophil group than in the BTS group. Fewer patients in the eosinophil group than in the BTS group were admitted to hospital because of asthma exacerbations ( $1 v 6$, $\mathrm{p}=0.047$ ). The groups did not differ for average daily dose of inhaled or oral corticosteroids.

\section{Conclusion}

In patients with asthma, a management strategy that controls lower airway eosinophilic inflammation and symptoms was more effective than standard care for reducing asthma exacerbations.

*See glossary.

†Information provided by author.

\section{COMMENTARY}

Most physicians agree that inflammation plays a pivotal role in the pathophysiology of asthma. Unfortunately, few agree on how this inflammation is to be detected and quantified. Fewer still agree about its prognostic or therapeutic significance. For instance, Warke $e t a l^{1}$ and Van Den Toorn $e t a l^{2}$ have shown airway eosinophilia during remission in patients with asthma and suggest that some other mediator of inflammation causes symptoms of asthma. However, they can only speculate on the long term clinical importance of their findings.

In the trial by Green et al, sputum eosinophil counts are used not just as a marker of inflammation but also as a predictor of clinical outcomes. The authors' supposition is that increasing eosinophilia in the airways precedes worsening of symptoms and that interventions based on responding to this finding will be beneficial to the patient. Their data support this supposition but are somewhat confusing. Although the eosinophil group had fewer severe exacerbations and fewer rescue courses of oral corticosteroids, the groups did not differ for several outcomes, including visual analogue symptom scores, total asthma quality of life scores, $\mathrm{FEV}_{1}$ after bronchodilator use, use of $\beta_{2}$ agonists, and mean dose of inhaled or oral steroids. Of particular note, $35 \%$ of patients in the eosinophil group had normal eosinophil counts for the entire 12 months of the study. Although the authors offer some speculation for the dissociation of inflammation from airflow obstruction and symptoms, our understanding of these processes has not advanced sufficiently to provide satisfactory answers.

Most current treatment protocols are based on the frequency and severity of patients' symptoms. ${ }^{3}$ As we have seen in several other disciplines, such as atherosclerotic cardiovascular disease, early intervention to prevent symptoms seems to be considerably more successful than reacting to symptoms. Studies such as this one are hopefully setting the groundwork for earlier and more definitive interventions.

Bernard Adelsberg, MD Connecticut Medical Group New Haven, Connecticut, USA

1 Warke TJ, Fitch PS, Brown V, et al. Outgrown asthma does not mean no airways inflammation. Eur Respir J 2002;19:284-7.

van den Toorn LM, Overbeek SE, de Jongste JC, et al. Airway inflammation is present during clinical remission of atop asthma. Am J Respir Crit Care Med 2001;164:2107-13
Guidelines for the Diagnosis and Management of Guidelines for the Diagnosis and Management of Asthma. Clinical Practice Guidelines. NIH Publication No 97-4051. 1997.
Source of funding: NHS Regional For correspondence: Dr I D Pavord, Glenfield Hospital, Leicester, UK. ian.Pavord@ uhl-tr.nhs.uk

Abstract and commentary also appear in ACP Journal Club. 